\title{
Estimativa da Forma de Lançamento de Poluentes em Cursos Hídricos Empregando Expansões em Autofunções e Inferência Bayesiana
}

\section{Estimation of the Pollutants Launch Form in Water Courses Employing Expansions Autofunctions and Bayesian Inference}

Bruno Carlos Lugão1, Diego Campos Knupp², Pedro Paulo Gomes Watts Rodrigues ${ }^{3}$, Antônio José da Silva Neto ${ }^{4}$, Luiz Alberto da Silva Abreu ${ }^{5}$

\section{RESUMO}

O presente trabalho tem como objetivo estimar uma função que representa o aporte de um agente poluidor em um rio. O problema direto é resolvido com o método híbrido analítico-numérico conhecido como Técnica da Transformada Integral Generalizada (GITT). Já o problema inverso é formulado via inferência bayesiana e resolvido através o Método de Monte Carlo via Cadeias de Markov (MCMC). Os resultados obtidos apresentam custo computacional relativamente baixo, além de um baixo erro relativo para as massas de poluente recuperadas.

Palavras-chave: Transformada integral, problema inverso, série de Fourier, cadeias de Markov.

\section{ABSTRACT}

The present work is aimed at estimating a timewise varying function that represents the release of a pollutant into a river. The direct problem is solved with the analyticalnumerical hybrid method known as the Generalized Integral Transformation Technique (GITT). The inverse problem is formulated employing the Bayesian framework and solved through the Markov Chains Monte Carlo method (MCMC). The solution demands relatively low computational cost, and present estimates with low relative error for the recovered pollutant masses.

Keywords: Integral transform, inverse problem, Fourier series and Markov chain.
${ }^{1}$ Universidade do Estado do Rio de Janeiro, Instituto Politécnico Nova Friburgo, RJ, Brasil.

E-mail: blugao@iprj.uerj.br

${ }^{2}$ Universidade do Estado do Rio de Janeiro, Instituto Politécnico Nova Friburgo, RJ, Brasil.

E-mail: diegoknupp@iprj.uerj.br

${ }^{3}$ Universidade do Estado do Rio de Janeiro, Instituto Politécnico Nova Friburgo, RJ, Brasil.

E-mail: pwatts@iprj.uerj.br

${ }^{4}$ Universidade do Estado do Rio de Janeiro, Instituto Politécnico Nova Friburgo, RJ, Brasil.

E-mail: ajsneto@iprj.uerj.br

${ }^{5}$ Universidade do Estado do Rio de Janeiro, Instituto Politécnico Nova Friburgo, RJ, Brasil.

E-mail: luiz.abreu@iprj.uerj.br 


\section{INTRODUÇĀOO}

A manutenção da qualidade dos meios hídricos é vista atualmente como um dos grandes desafios da humanidade, uma vez que a água é vital para a manutenção da vida não somente do homem, mas de todos os seres vivos presentes na natureza. Dessa maneira, se faz necessário o desenvolvimento de ações voltadas para preservação deste recurso para a atual e as futuras gerações.

Nesse contexto, o desenvolvimento de estudos relacionados às atividades que possuem risco de acarretar algum impacto ao meio ambiente, bem como o monitoramento dos padrões de qualidade da água exigidos, se tornam importantes ferramentas no processo de tomada de decisão pelos órgãos de gestão ambiental. Muitas vezes, esses estudos se tornam onerosos por requererem o intensivo emprego de material e pessoal especializado. Por consequência, o desenvolvimento de modelos matemáticos e computacionais no gerenciamento de recursos hídricos se torna uma alternativa interessante face às limitações financeiras existentes. De acordo com Lima (2012), essas metodologias são ferramentas adequadas para o diagnóstico e prognóstico em ambientes aquáticos, visto que, uma vez calibrados, diversos cenários podem ser produzidos, conforme os dados de entrada sejam manipulados.

O objetivo principal deste trabalho é, através da solução de um problema inverso, identificar de maneira aproximada o modo com que um determinado poluente é lançado em um hipotético curso fluvial. A solução do problema direto é realizada através da Técnica da Transformada Integral Generalizada (GITT) (COTTA, 1993). Enquanto que a análise inversa visa estimar uma função, que representa a condição de contorno na fronteira a montante do domínio, estimativa essa que é conduzida por meio da determinação dos coeficientes de uma série de Fourier.

Existem diversos trabalhos na literatura que analisam a dispersão de poluentes em rios, encontrando-se diferentes abordagens, tanto na resolução do problema direto quanto na análise inversa (Hamdi (2009), Wang (2012), Verdière (2013), Parolin (2015), Li (2016), Kulbay (2016) e Lugão(2017)). Estas pesquisas envolvem a recuperação de fontes de poluição em bacias hidrográficas, considerando domínios finitos e infinitos, a estimativa de parâmetros como localização, intensidade e duração do despejo de efluentes cuja forma funcional é considerada conhecida a priori, além de estimativas de funções que representam a maneira com que um determinado poluente é inserido no meio hídrico. 


\section{FORMULAÇAOO E SOLUÇAOO DO PROBLEMA DIRETO}

O lançamento de um dado poluente em um curso fluvial, suficientemente estreito e raso para que se justifique uma abordagem integrada na seção transversal, pode convenientemente ser modelado pela equação (Fischer, 1979):

$$
\begin{gathered}
\frac{\partial C(x, t)}{\partial t}+u \frac{\partial C(x, t)}{\partial x}=E_{L} \frac{\partial^{2} C(x, t)}{\partial x^{2}}, \quad 0<x<L_{x} \text { e } t>0 \\
C(0, t)=g(t), \quad t>0 \\
C(L, t)=0, \quad t>0 \\
C(x, 0)=f(x), \quad 0<x<L_{x}
\end{gathered}
$$

onde $E_{L}$ é o coeficiente de dispersão longitudinal, ué a velocidade média do escoamento, $L_{x}$ é o comprimento da seção do rio analisada, as eqs (1.b) e (1.c) são as condições de contorno e a eq (1.d) a condição inicial. Na figura 1 tem-se a representação esquemática para o lançamento de um poluente em um rio, onde $Q_{R}$ é a vazão e $x_{\text {exp }}$ é a posição de coleta dos dados experimentais localizada a jusante do local de despejo.

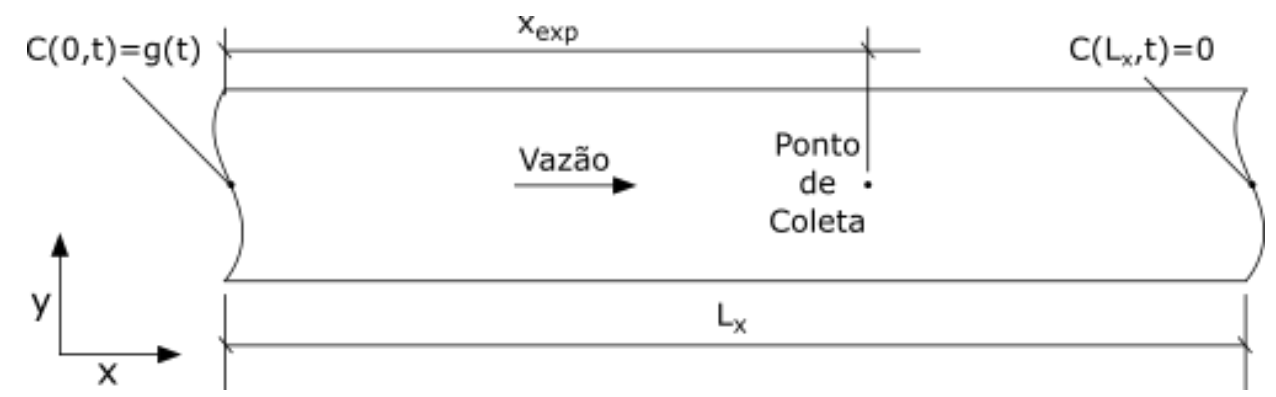

Figura 1. Representação Esquemática do Problema Direto

Neste trabalho, para a solução do problema direto, foi utilizada a Técnica da Transformada Integral Generalizada na resolução da eq. (1). Esta metodologia foi desenvolvida por Cotta (1993) e compreende uma extensão da Técnica da Transformada Integral Clássica proposta por Ozisik (1984). A GITT é um método híbrido numéricoanalítico que emprega uma base de autofunções obtida a partir de um problema auxiliar de autovalor. Uma vez conhecidas as autofunções e seus respectivos autovalores é aplicado um operador de transformação integral que permite obter um sistema de equações diferenciais ordinárias. Este sistema é truncado em um determinado número de termos, suficientes para que a precisão requerida seja alcançada, já que geralmente é possível resolvê-lo apenas por meio de abordagens numéricas.

Observa-se que a eq. (1) é não homogênea. Ao se utilizar a Técnica da 
Transformada Generalizada, como apontado por Cotta (1997), sempre é interessante diminuir a importância dos termos fontes e das condições de contorno de modo a melhorar a convergência. Com o intuito de contornar esta dificuldade foi utilizado um filtro capaz de diminuir a influência do contorno na solução proposta. Suponha que o potencial desejado, $C(x, t)$, possa ser escrito como a soma de um potencial filtrado, $C^{*}(x, t)$, com um filtro, $C_{f}(x, t)$, ou seja:

Sendo

$$
C(x, t)=C^{*}(x, t)+C_{f}(x, t)
$$

$$
C_{f}(x, t)=\frac{g(t)}{e^{\frac{u u_{x}}{E_{L}}-1}}\left(e^{\frac{u L_{x}}{E_{L}}}-e^{\frac{u x}{E_{L}}}\right)
$$

O problema de autovalor diferencial é definido como o problema Sturm Liouville Cotta (1993), cujas autofunções, $\psi_{i}(x)$, e seus respectivos autovalores, $\mu_{i}$, para as condições de contorno de primeiro tipo homogêneas são:

$$
\begin{gathered}
\psi_{i}(x)=\sin \left(\frac{\mu_{i} x}{\sqrt{E_{L}}}\right), \quad i=1,2, \ldots \\
\mu_{i}=\frac{i \pi \sqrt{E_{L}}}{L_{x}}, \quad i=1,2, \ldots
\end{gathered}
$$

Agora é possível definir o par de transformação integral como:

$$
\begin{aligned}
& \text { Transformada: } \bar{C}_{i}(t)=\int_{0}^{L_{x}} \tilde{\psi}_{i}(x) C^{*}(x, t) d x \\
& \text { Inversa: } C^{*}(x, t)=\sum_{i=1}^{\infty} \bar{C}_{i}(t) \tilde{\psi}_{i}(x)
\end{aligned}
$$

sendo $\bar{C}_{i}(t)$ os potenciais transformados e $\tilde{\psi}_{i}(x)$ as autofunções normalizadas, cuja expressão é dada por:

$$
\tilde{\psi}_{i}(x)=\frac{\psi_{i}(x)}{\sqrt{N_{i}}}, \quad N_{i}=\int_{0}^{L_{x}} \psi_{i}^{2}(x) d x
$$

Utilizando o operador $\int_{0}^{L_{x}}(.) \tilde{\psi}_{i}(x) d x$ no problema filtrado e realizando manipulações algébricas sobre cada termo resultante, como pode ser visto em Cotta (1993), obtém-se o seguinte sistema de EDO's para o potencial transformado $\overline{C_{i}}(t)$ :

$$
\frac{d \bar{C}_{i}(t)}{d t}+\mu_{i}^{2} \bar{C}_{i}(t)=\bar{g}_{i}(t), \quad i=1,2,3, \ldots
$$


Sendo

$$
\bar{g}_{i}(t)=-u \sum_{j=1}^{\infty} \bar{C}_{j}(t) D_{i j}-G_{i}
$$

$$
D_{i j}=\int_{0}^{L_{x}} \tilde{\psi}_{i}(x) \frac{d \tilde{\psi}_{j}(x)}{d x} d x \text { e } G_{i}=\int_{0}^{L_{x}} \tilde{\psi}_{i}(x) \frac{\partial C_{f}(x, t)}{\partial t} d t
$$

com as condições iniciais transformadas dadas por:

$$
\bar{C}_{i}(0)=\int_{0}^{L_{x}} \tilde{\psi}_{i}(x) f^{*}(x) d x
$$

O sistema de eqs. diferencias ordinárias definido acima não possui uma solução analítica, portanto se faz necessário truncá-lo em uma determinada ordem, Ntr, suficientemente grande para que a precisão desejada seja alcançada com a utilização de algum método numérico. Para a resolução do sistema definido pela eq. (8) foi empregada a rotina NDSolve do software Mathematica.

Uma vez conhecidos os potenciais transformados, $\bar{C}_{i}(t)$, e as autofunções normalizadas, $\tilde{\psi}_{i}(x)$, é possível utilizar a fórmula da transformada inversa eq. (6.b) para escrever o potencial filtrado $C^{*}(x, t)$. Dessa maneira, a solução do problema definido pela eq. (1) pode ser representada como:

$$
C(x, t)=C^{*}(x, t)+C_{f}(x, t)=\sum_{i=1}^{N t r} \bar{C}_{i}(t) \tilde{\psi}_{i}(x)+C_{f}(x, t)
$$

\section{FORMULAÇAO E SOLUÇAOO DO PROBLEMA INVERSO}

O problema inverso aqui estudado consiste em estimar a função $g(t)$, dada pela eq. (1.b), que representa o despejo de um poluente conservativo em um hipotético curso fluvial, a partir de um conjunto de dados experimentais sintéticos colhidos em uma posição de interesse localizada a jusante do local de lançamento. Para tanto, os valores da velocidade média do escoamento e o coeficiente de dispersão longitudinal foram considerados conhecidos e constantes.

Neste trabalho, a função $g(t)$ foi aproximada por uma série de Fourier constituída apenas por cossenos da seguinte forma:

$$
g(t) \cong \frac{a_{0}}{2}+\sum_{n=1}^{k} a_{n} \cos \left(\frac{n \pi x}{t_{f}}\right)
$$

onde $t_{f}$ é o tempo final da simulação e $a_{n}$ representa os coeficientes de Fourier. Esta metodologia foi escolhida visando diminuir a quantidade de parâmetros a serem 
estimados, de modo a se reduzir o custo computacional envolvido na estimativa.

$\mathrm{Na}$ abordagem Bayesiana o problema inverso é formulado como um problema de inferência estatística, de maneira que se possa incorporar toda a informação disponível ao modelo, visando a redução do nível de incerteza presente na análise inversa. O teorema de Bayes para problemas inversos compreende todos os princípios da inversão estatística citados por Kaipio (2004), sendo expresso como:

$$
\pi_{p o s}(\mathbf{Z})=\pi(\mathbf{Z} \mid \mathbf{Y})=\frac{\pi_{p r}(\mathbf{Z}) \pi(\mathbf{Y} \mid \mathbf{Z})}{\pi(\mathbf{Y})}
$$

onde $\mathbf{Z}=\left\{a_{1}, a_{2}, a_{3}, \ldots, a_{i}, \ldots, a_{k}\right\}$ é o vetor de parâmetros contendo os coeficientes de Fourier, $\mathbf{Y}=\left\{y_{1}, y_{2}, y_{3}, \ldots, y_{\text {nexp }}\right\}$ representa o vetor de medidas experimentais, $\pi_{\text {pos }}(\mathbf{Z})$ é a distribuição a posteriori, $\pi(\mathbf{Y} \mid \mathbf{Z})$ é a verossimilhança, $\pi_{p r}(\mathbf{Z})$ é a distribuição a priori e $\pi(\mathbf{Y})$ é a densidade de probabilidade marginal, que exerce um papel de constante de normalização.

Assumindo que os erros experimentais são aditivos, não correlacionados e modelados por uma distribuição normal, a verossimilhança pode ser expressa como:

$$
\pi(\mathbf{Y} \mid \mathbf{Z})=\frac{1}{\sqrt{(2 \pi)^{\text {nexp }}}} \frac{1}{\operatorname{det}(\mathbf{W})} \exp \left[-\frac{1}{2}\left(\mathbf{Y}-\mathbf{Y}_{\text {calc }}\right)^{\mathrm{T}} \mathbf{W}^{-1}\left(\mathbf{Y}-\mathbf{Y}_{\text {calc }}\right)\right]
$$

sendo Wa matriz de covariância dos dados experimentais, nexpé o número de medidas e $\mathbf{Y}_{\text {calc }}$ o vetor com os valores das concentrações calculadas de acordo com a GITT.

Neste trabalho, para se obter uma aproximação da distribuição a posteriori, será utilizado o Método de Monte Carlo com Cadeias de Markov (MCMC) para a resolução do problema inverso, implementado de acordo com o algoritmo de Metropolis-Hastings, utilizando uma distribuição normal como densidade de transição.

\section{RESULTADOS E DISCUSSAO}

Nesta seção serão apresentados os principais resultados para a estimativa de $g(t)$ utilizando uma aproximação por série de Fourier constituída apenas por cossenos. Para tanto, foram utilizados dados experimentais sintéticos calculados através da GITT, empregando uma alta ordem de truncamento. Quanto ao ruído experimental, este foi simulado através de uma distribuição normal com média zero e desvio padrão igual a uma determinada porcentagem $(5 \%$ ou $2,5 \%)$ da concentração de pico da pluma de 
contaminantes no ponto de amostragem. Cabe destacar que, para o fenômeno em análise, foi considerando $L_{x}=400 \mathrm{~m}, u=0.59 \mathrm{~m} / \mathrm{s}, E_{L}=1.89 \mathrm{~m}^{2} / \mathrm{s}, Q_{R}=538 \mathrm{l} / \mathrm{s}$, sendo os dados tomados na posição $x=150 \mathrm{~m}$ durante o intervalo de tempo de $0 \leq t \leq 1200 \mathrm{~s}$ , a, aproximadamente, cada 2,4 s.

Segundo Kaipio (2004), ao fazer uso de dados simulados gerados pelo mesmo modelo empregado na análise inversa ocorre o que este autor chama de crime inverso. Este processo consiste em tornar o problema menos mal posto do que ele realmente é, conduzindo assim, a conclusões enganosas. De maneira a diminuir a presença do crime inverso no presente trabalho, foi adotada a metodologia aplicada por Knupp (2015), onde os dados experimentais foram gerados com uma ordem de truncamento $(\mathrm{Ntr})$ muito maior do que aquela utilizada na resolução do problema inverso.

O tipo de lançamento de poluente considerado neste estudo representa dois despejos contínuos de duração finita com intensidades distintas, sendo modelado pela seguinte expressão:

$$
g(t)=\frac{C_{1}}{1+\exp \left(\gamma\left(t-\tau_{1}\right)\right)+\exp \left(\gamma\left(\tau_{2}-t\right)\right)}+\frac{C_{2}}{1+\exp \left(\gamma\left(t-\tau_{3}\right)\right)+\backslash \exp \left(\gamma\left(\tau_{4}-t\right)\right)}+C_{0}
$$

onde $C_{1}$ e $C_{2}$ são as concentrações do poluente, $\tau_{1}$ e $\tau_{2}$ indicam o intervalo de tempo que $C_{1}$ foi lançado $\tau_{1} \leq t \leq \tau_{2}$, assim como $\tau_{3}$ e $\tau_{4}$ representam o intervalo de tempo do derramamento de $C_{2} \tau_{3} \leq t \leq \tau_{4}, C_{0}$ é a concentração de poluente já existente no rio e $\gamma$ é um parâmetro que controla a suavidade das variações abruptas de concentração. Os valores dos coeficientes da eq. (13) utilizados para gerar as medidas experimentais sintéticas são dados na tabela 1 .

Tabela 1.Valores dos coeficientes da função $g(t)$

\begin{tabular}{cccccccc}
\hline$C_{1}[\mathrm{mg} / \mathrm{l}]$ & $\tau_{1}[s]$ & $\tau_{2}[s]$ & $C_{2}[\mathrm{mg} / \mathrm{l}]$ & $\tau_{3}[s]$ & $\tau_{4}[s]$ & $C_{0}[\mathrm{mg} / l]$ & $\gamma$ \\
\hline 100 & 100 & 300 & 50 & 500 & 700 & 15,5 & 0,5 \\
\hline
\end{tabular}

A solução do problema inverso foi formulado computacionalmente no software de computação algébrica Mathematica, utilizando cadeias de Markov com 80.000 amostras e um aquecimento de 40.000 estados, empregando uma ordem de truncamento $\mathrm{Ntr}=50$ para a Técnica da Transformada Integral Generalizada. Na tabela 2 é possível analisar a convergência da GITT, utilizando a aproximação de $g(t)$ por uma série de Fourier com 20 termos como condição de contorno da eq. (1). Observando o comportamento da solução 
a medida que a ordem de truncamento aumenta, percebe-se que esta abordagem chega a alcançar cinco dígitos de convergência para $\mathrm{Ntr}=200$.

Tabela 2. Estudo da convergência para a solução do problema direto utilizando GITT

\begin{tabular}{cccccccccc}
\hline & \multicolumn{3}{c}{$t=60 \mathrm{~s}$} & \multicolumn{3}{c}{$t=180 \mathrm{~s}$} & \multicolumn{3}{c}{$t=300 \mathrm{~s}$} \\
Ntr & $50 \mathrm{~m}$ & $100 \mathrm{~m}$ & $150 \mathrm{~m}$ & $50 \mathrm{~m}$ & $100 \mathrm{~m}$ & $150 \mathrm{~m}$ & $50 \mathrm{~m}$ & $100 \mathrm{~m}$ & $150 \mathrm{~m}$ \\
\hline 10 & 17,397 & 63,867 & 118,746 & 15,220 & 17,236 & 95,153 & 15,211 & 13,979 & 27,807 \\
25 & 15,929 & 64,323 & 116,814 & 15,628 & 14,118 & 93,651 & 15,398 & 16,730 & 30,197 \\
50 & 16,091 & 64,004 & 116,569 & 15,473 & 15,233 & 94,242 & 15,489 & 15,766 & 29,713 \\
75 & 16,091 & 63,894 & 116,521 & 15,502 & 14,960 & 94,103 & 15,511 & 15,623 & 29,636 \\
100 & 16,097 & 63,846 & 116,496 & 15,501 & 14,979 & 94,112 & 15,505 & 15,672 & 29,662 \\
125 & 16,100 & 63,823 & 116,483 & 15,497 & 15,019 & 94,132 & 15,499 & 15,719 & 29,686 \\
150 & 16,100 & 63,812 & 116,478 & 15,502 & 14,983 & 94,113 & 15,498 & 15,730 & 29,691 \\
175 & 16,102 & 63,805 & 116,475 & 15,500 & 15,000 & 94,122 & 15,500 & 15,720 & 29,686 \\
200 & 16,102 & 63,803 & 116,473 & 15,500 & 14,999 & 94,121 & 15,500 & 15,710 & 29,681 \\
\hline
\end{tabular}

Na figura 2 é possível comparar o comportamento da função utilizada para simular - lançamento do poluente com aquele apresentado pela função que foi estimada, expressa aqui por uma aproximação por série de Fourier com 20 termos, tendo sido adotados valores exatos para os coeficientes. É importante ressaltar a dificuldade encontrada pelo MCMC na estimativa de $\$ g(t) \$$ para os tempos $t \leq 1000 \mathrm{~s}$. Este problema se deve ao fato de que, a partir deste instante de tempo, a pluma de contaminantes não poder ser detectada por completo na posição definida como o ponto de amostragem, sendo esta, portanto, uma limitação física do problema.

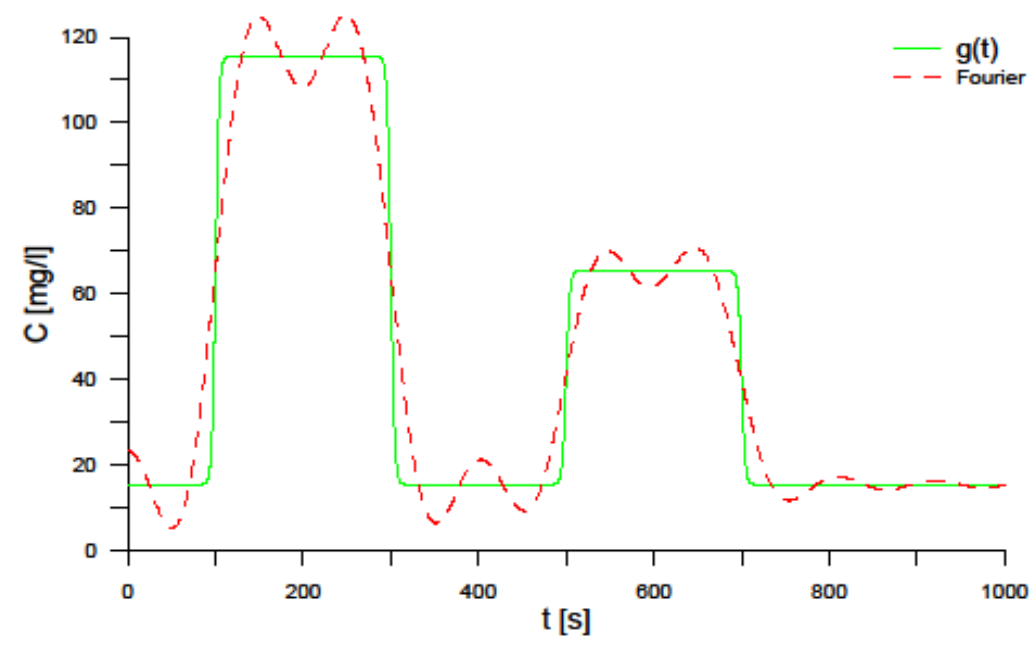

Figura 2. Aproximação de $g(t)$ por uma série de Fourier. 
A figura 3 apresenta os resultados obtidos para a determinação da condição de contorno da eq. da advecção-dispersão, dada pela expressão (1b), por meio da estimativa dos valores dos coeficientes de uma série de Fourier. Em (a),(c) e (e) encontram-se os resultados utilizando o conjunto de dados com erro $\sigma_{\text {exp }}=5,52 \mathrm{mg} / \mathrm{l}$, enquanto que nos gráficos (b), (d) e (f) tem-se os resultados para o conjunto de dados com erro $\sigma_{\text {exp }}=$ $2,76 \mathrm{mg} / \mathrm{l}$. Em (a) e (b) é possível comparar a função $g(t)$, representada em azul e a aproximação por Fourier em vermelho. Observa-se que foi encontrado um bom ajuste entre o exato e o estimado, mesmo na presença de quatro variações abruptas nos valores de $g(t)$ para os dois casos. Já os gráficos (c) e (d) expõem o ajuste entre a solução do problema direto, utilizando a função recuperada como condição de contorno em $x=0 \mathrm{~m}$ na eq. (1), e os dados experimentais sintéticos. Foi obtido um erro médio quadrático de 5,68032 e 2,72139 e um erro médio relativo para as massas recuperadas de 0,38\% e $0,57 \%$, respectivamente para os conjuntos com desvios experimentais de $5 \%$ e $2,5 \%$ da concentração de pico dos dados exatos. Este resultado encontra-se dentro do esperado, uma vez que os valores do RMS se aproximaram dos desvios experimentais considerados. Nos gráficos (e) e (f), tem-se os resíduos calculados entre a resposta do modelo e as medidas experimentais. Observa-se um comportamento aleatório em torno da reta $C=0$, sendo este resultado condizente com a hipótese assumida dos erros experimentais serem considerados não correlacionados e modelados por uma distribuição normal de média zero e desvio padrão constante.

Devido à quantidade de parâmetros presentes no problema inverso, serão exibidas as cadeias de Markov apenas para os coeficientes $a_{5}$ e $a_{10}$ da série de Fourier. A figura 4 apresenta a evolução das cadeias de Markov para os parâmetros anteriormente citados, onde (a)-(b) e (c)-(d) representam, respectivamente, a solução para o conjunto de dados com erro experimental maior e menor. Claramente as cadeias não foram capazes de alcançar o estado de equilíbrio com o número de amostras estipulado, apesar do bom ajuste encontrado para a função recuperada e para a os dados experimentais. Cabe ressaltar que não foi utilizado qualquer tipo de informação a priori acerca dos coeficientes que se busca estimar, o que dificulta ainda mais a solução devido a natureza mal posta do problema. 


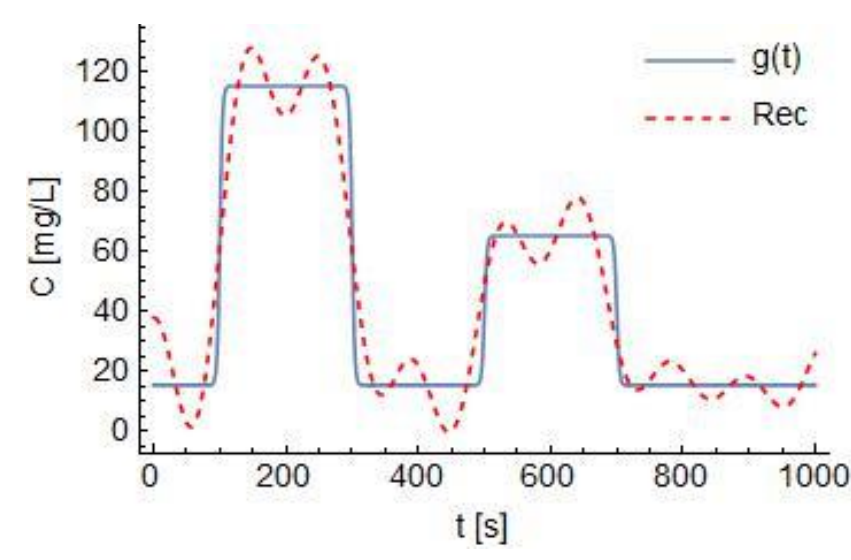

(a)

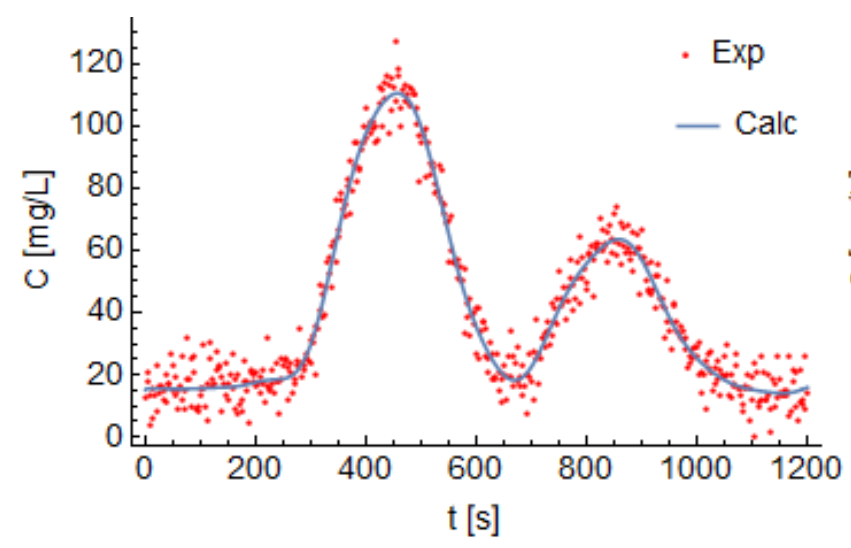

(c)

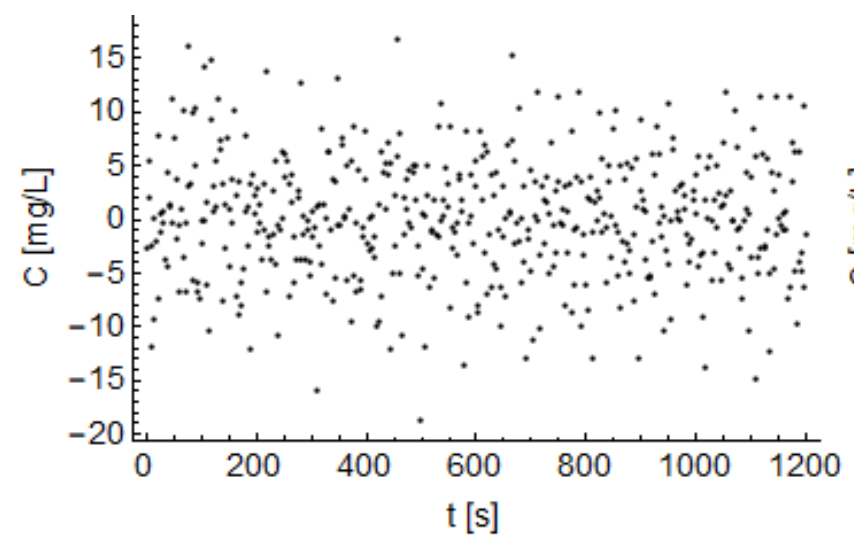

(e)

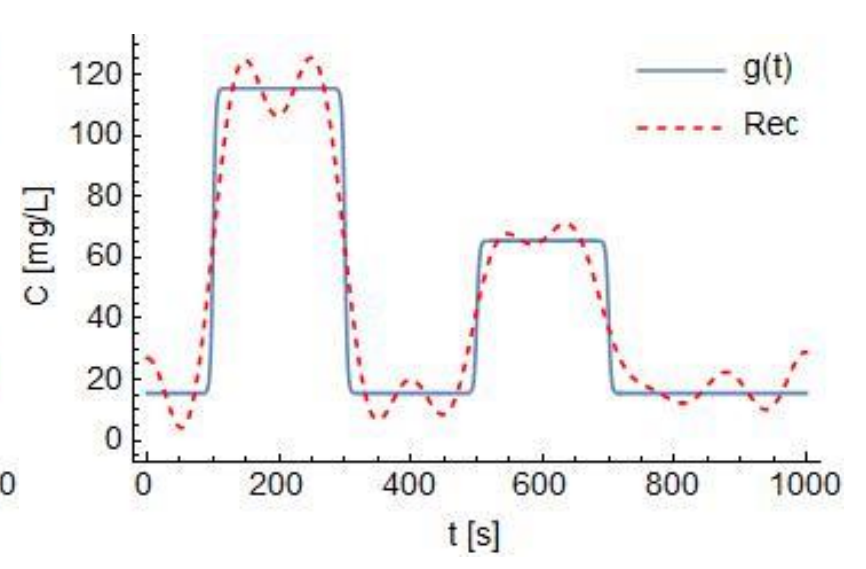

(b)

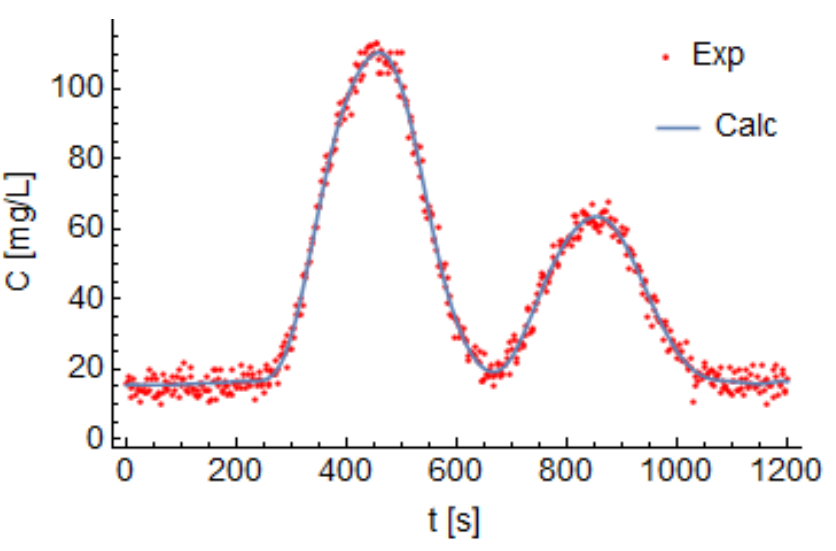

(d)

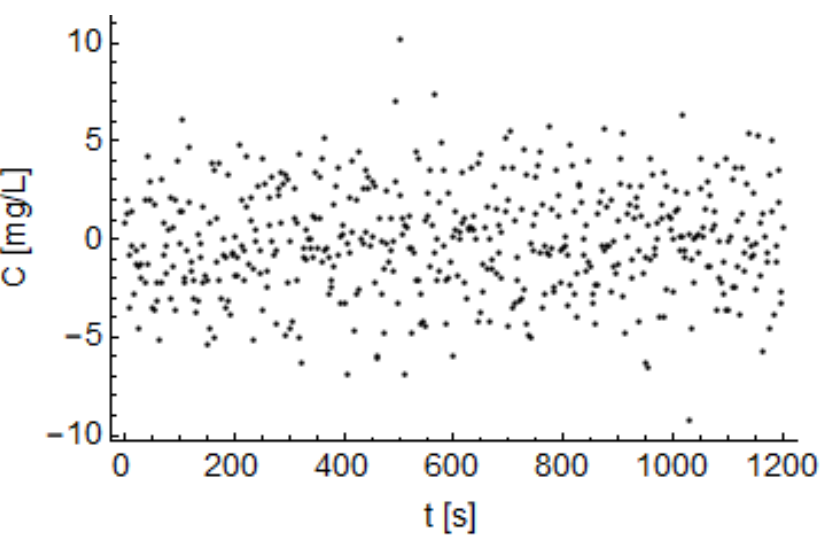

(f)

Figura 3. Resultados para a estimativa de $g(t)$ através de uma série de Fourier com 20 termos, utilizando um conjunto de dados experimentais sintéticos com erro $\sigma_{\text {exp }}=$ $5,52 \mathrm{mg} / \mathrm{l}$ em (a), (c) e (e) e $\sigma_{\text {exp }}=2,76 \mathrm{mg} / \mathrm{l}$ em (b), (d) e (f).

Um último ponto que merece destaque é a significativa redução do custo computacional do problema inverso ao se aproximar $g(t)$ por uma série de Fourier em comparação com os resultados obtidos por Lugão (2017), que utilizou uma interpolação linear para resolver o mesmo problema. Esta metodologia apresentou uma diminuição de 
aproximadamente $96 \%$ na execução de todo o processo iterativo do MCMC, reduzindo o tempo utilizado na solução do problema inverso de 62 horas para 3 horas.

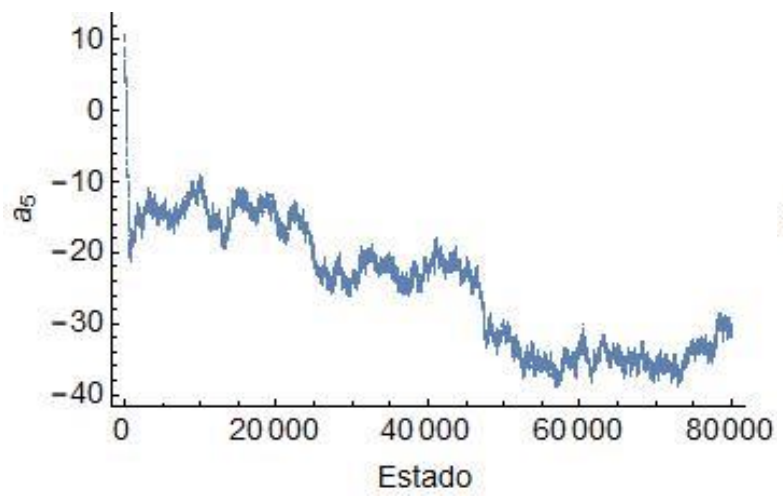

(a)

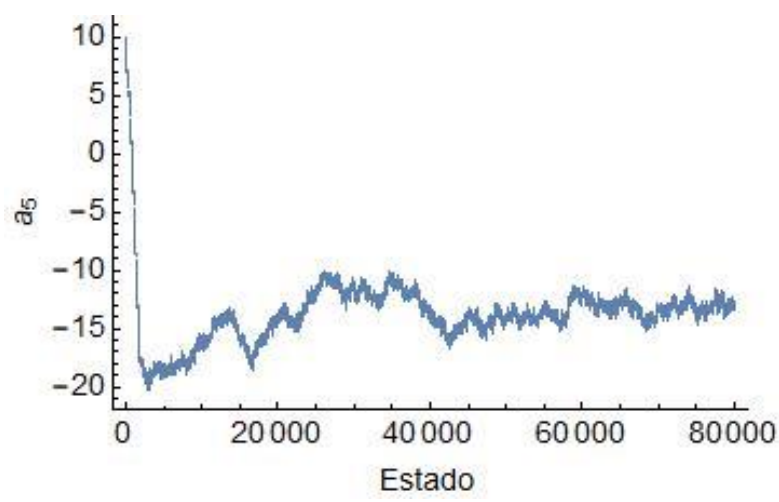

(c)

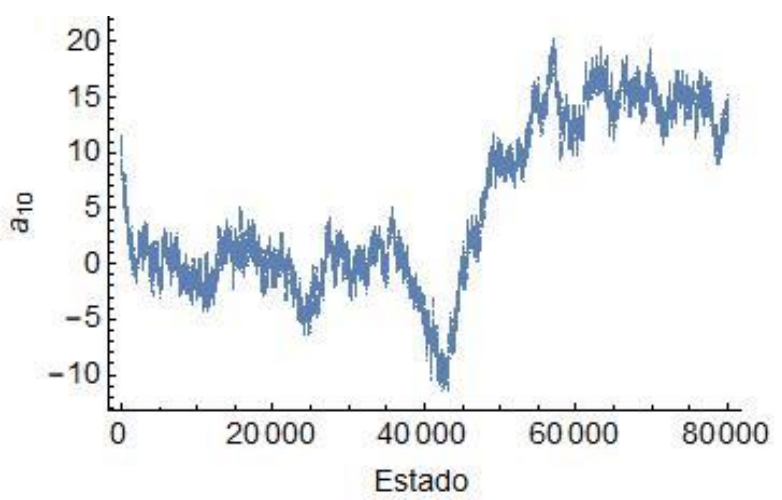

(b)

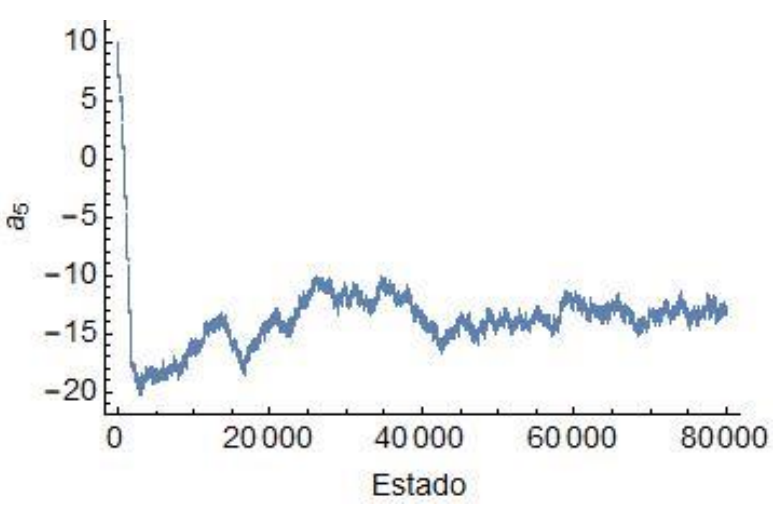

(d)

Figura 4. Cadeias de Markov para os coeficientes $a_{5}$ e $a_{10}$.

\section{DISCUSSAOO}

Este trabalho teve como objetivo principal a estimativa de uma função que pudesse expressar uma possível de contorno da eq. da advecção-dispersão unidimensional em regime transiente. Para tanto, utilizou-se a Técnica da Transformada Integral Generalizada na resolução do problema direto e do Método de Monte Carlo com Cadeias de Markov na formulação do problema inverso, sem o uso de qualquer informação a priori. A metodologia proposta foi capaz de recuperar de maneira satisfatória a forma como hipoteticamente se daria o lançamento desse poluente em um curso fluvial idealizado, mesmo com os baixos níveis de informação utilizados.

Outro aspecto importante a ser destacado foi a dificuldade em se estimar $g(t)$ para os tempos $t \leq 1000 \mathrm{~s}$ no problema inverso. Isso se deve ao fato de que a pluma de contaminante, para o intervalo de tempo considerado, não ser detectada por completo na 
posição definida como o ponto de monitoramento. Pode-se entender que esta é uma limitação física do problema estudado, já a inexistência de dados experimentais inviabiliza a própria estimativa neste caso.

Por fim, a metodologia proposta apresentou uma significativa redução do custo computacional, se mostrando apropriada para a estimativa de funções que compreendem a condição de contorno a montante do domínio simulado, respeitadas as condições necessárias para a utilização do modelo unidimensional. A modelagem empregada possui potencial de aplicação na gestão de recursos hídricos, seja na tomada de decisões ou na identificação de possíveis responsáveis por danos ambientais.

\section{AGRADECIMENTOS}

Os autores agradecem pelo apoio financeiro das agências CNPq, CAPES e FAPERJ.

\section{REFERÉNCIAS}

Cotta, R. M. Integral transforms in computational heat and fluid flows. Florida, CRC Press, 1993.

Cotta, R. M.; Mikhailov, M. D. Heat conduction: lumped analysis, integral transforms, symbolic computation. John Wiley \& Son Limited, 1997.

Fischer, H. B.; List, J. E.; Koh, C. R.; Imberger J.; Brooks, N. H. Mixing in inland and coastal waters. Academic Press, London, 1979.

Hamdi, A. The recovery of a time-dependent point source in a linear transport equation: application to surface water pollution. Inverse Problems, v 25, n 7, 2009.

Kaipio, J., Sommersalo, E.Statistical and Computational Inverse Problems, SpringerVerlag, 2004.

Knupp, D. C.; Sacco, W. F.; Slva Neto, A. J. Direct and inverse analysis of diffusive logistic population evolution with time delay and impulsive culling via integral transforms and hybrid optimization. Applied Mathematics and Computation, v 221, 105-120, 2015.

Kulbay, M.; Mukanova, B.; Sebu, C. Identification of separable sources for advectiondiffusion equations with variable diffusion coefficient from boundary measured data. Inverse Problems in Science and Engineering, 2016.

Li, Z.; Mao, X. Z.; Li, T. S.; Zhang, S. Estimation of river pollution source using the spacetime radial basis collocation method. Advances in Water Resources, v 88, 2016. 
Lugão, B.C.Estimativa de Fontes de Poluição em Meio Hídrico com Variação Temporal via Transformações Integrais e Método de Monte Carlo com Cadeias de Markov. Dissertação(Mestrado) — IPRJ/UERJ, Nova Friburgo, 2017.

Ozisik, M. N.; Mikhailov, M. D.Unified analysis and solutions of heat and mass diffusion.John Wiley, New York, 1984.

Parolin, R. S.; Rodrigues, P. P. G. W.; Silva Neto, A. J. Adaptação e aplicação de métodos de inteligência computacional para a estimação de uma fonte de poluentes em estuários. ENGEVISTA, v 17, n 2, 2015.

Verdière, N.; Joly-Blanchard, G.; Denis-Vidal, L. Identifiability and identification of a pollution source in a river by using a semi-discretized model. Applied Mathematics and Computation, v 221, 2013.

Wang, Z.; Liu, J. Identification of the pollution source from one-dimensional parabolic equation models. Applied Mathematics and Computation, v 219, n 8, 2012. 